\title{
How ObGyns can best work with radiologists to optimize screening for patients with dense breasts
}

\author{
Discussions with patients around breast cancer screening typically rest \\ on the shoulders of ObGyns. When a patient is informed she has dense \\ breasts, what should your dialogue include, and what resources are \\ available to you?
}

Elizabeth Etkin-Kramer, MD; DaCarla M. Albright, MD; and JoAnn Pushkin

\section{IN THIS} ARTICLE

Effective collaboration

page 21

\section{Reducing \\ barriers}

and cost

page 22

Pearls

for patient discussion

page 22 f your ObGyn practices are anything like ours, every time there is news coverage of a study regarding mammography or about efforts to pass a breast density inform law, your phone rings with patient calls. In fact, every density inform law enacted in the United States, except for in Illinois, directs patients to their referring provider-generally their ObGyn - to discuss the screening and risk implications of dense breast tissue.

The steady increased awareness of breast density means that we, as ObGyns and other primary care providers (PCPs), have additional responsibilities in managing the breast health of our patients. This includes guiding discus-

\footnotetext{
Dr. Etkin-Kramer is Assistant Professor, Florida International University School of Medicine, and Founder, Yodeah.org, Miami Beach, Florida.

Dr. Albright is Associate Professor, Associate Dean for Student Affairs and Wellness, University of Pennsylvania Perelman School of Medicine, Philadelphia, Pennsylvania.

Ms. Pushkin is Executive Director, DenseBreast-info.org.
}

Dr. Etkin-Kramer reports being an unpaid medical advisory board member for Bright Pink and the founder of Yodeah.org. Dr. Albright reports being a speaker for and serving on the medical advisory board for Hologic, Inc. Ms. Pushkin reports no financial relationships relevant to this article.

doi: 10.12788 /obgm.0040 sions with patients about what breast density means and whether supplemental screening beyond mammography might be beneficial.

As members of the Medical Advisory Board for DenseBreast-info.org (an online educational resource dedicated to providing breast density information to patients and health care professionals), we are aware of the growing body of evidence demonstrating improved detection of early breast cancer using supplemental screening in dense breasts. However, we know that there is confusion among clinicians about how and when to facilitate tailored screening for women with dense breasts or other breast cancer risk factors. Here we answer 6 questions focusing on how to navigate patient discussions around the topic and the best way to collaborate with radiologists to improve breast care for patients.

\section{Play an active role}

1. What role should ObGyns and PCPs play in women's breast health?

Elizabeth Etkin-Kramer, MD: I am a firm believer that ObGyns and all women's health providers should be able to assess their patients' risk of breast cancer and explain the process for managing this risk with 
their patients. This explanation includes the clinical implications of breast density and when supplemental screening should be employed. It is also important for providers to know when to offer genetic testing and when a patient's personal or family history indicates supplemental screening with breast magnetic resonance imaging (MRI).

DaCarla M. Albright, MD: I absolutely agree that PCPs, ObGyns, and family practitioners should spend the time to be educated about breast density and supplemental screening options. While the exact role providers play in managing patients' breast health may vary depending on the practice type or location, the need for knowledge and comfort when talking with patients to help them make informed decisions is critical. Breast health and screening, including the importance of breast density, happen to be a particular interest of mine. I have participated in educational webinars, invited lectures, and breast cancer awareness media events on this topic in the past.

\section{Join forces with}

\section{imaging centers}

\section{How can ObGyns and radiologists collaborate most effectively to use screening results to personalize breast care for patients?}

Dr. Etkin-Kramer: It is important to have a close relationship with the radiologists that read our patients' mammograms. We need to be able to easily contact the radiologist and quickly get clarification on a patient's report or discuss next steps. Imaging centers should consider running outreach programs to educate their referring providers on how to risk assess, with this assessment inclusive of breast density. Dinner lectures or grand round meetings are effective to facilitate communication between the radiology community and the ObGyn community. Finally, as we all know, supplemental screening is often subject to copays and deductibles per insurance coverage. If advocacy groups, who are working to eliminate these types of costs, cannot get insurers to waive these payments, we need a less expensive self-pay option.
Dr. Albright: I definitely have and encourage an open line of communication between my practice and breast radiology, as well as our breast surgeons and cancer center to set up consultations as needed. We also invite our radiologists as guests to monthly practice meetings or grand rounds within our department to further improve access and open communication, as this environment is one in which greater provider education on density and adjunctive screening can be achieved.

\section{Know when to refer \\ a high-risk patient}

\section{Most ObGyns routinely collect family history and perform formal risk assessment. What do you need to know about referring patients to a high-risk program?}

Dr. Etkin-Kramer: It is important as ObGyns to be knowledgeable about breast and ovarian cancer risk assessment and genetic testing for cancer susceptibility genes. Our patients expect that of us. I am comfortable doing risk assessment in my office, but I sometimes refer to other specialists in the community if the patient needs additional counseling. For risk assessment, I look at family and personal history, breast density, and other factors that might lead me to believe the patient might carry a hereditary cancer susceptibility gene, including Ashkenazi Jewish ancestry. ${ }^{1}$ When indicated, I check lifetime as well as shortterm (5- to 10-year) risk, usually using Breast Cancer Surveillance Consortium (BCSC) or Tyrer-Cuzick/International Breast Cancer Intervention Study (IBIS) models, as these include breast density.

I discuss risk-reducing medications. The US Preventive Services Task Force recommends these agents if my patient's 5-year risk of breast cancer is $1.67 \%$ or greater, and I strongly recommend chemoprevention when the patient's 5-year BCSC risk exceeds $3 \%$, provided likely benefits exceed risks. ${ }^{2,3}$ I discuss adding screening breast MRI if lifetime risk by Tyrer-Cuzick exceeds $20 \%$. (Note that Gail and BCSC models are not recommended to be used to determine risk for purposes of

\begin{tabular}{l} 
FAST \\
TRACK \\
\hline Maintain close \\
relationships \\
with radiologists/ \\
imaging centers \\
so that your \\
questions can \\
be answered \\
quickly and \\
appropriately
\end{tabular}

\section{FAST}

Maintain close relationships with radiologists/ imaging centers so that your questions can appropriately 
FAST

TRACK

Be familiar
with the breast
screening
orders your
institution's
EHR offer; they
may be helpful
to avoid
additional
scripts down
the line

supplemental screening with MRI as they do not consider paternal family history nor age of relatives at diagnosis.)

Dr. Albright: ObGyns should be able to ascertain a pertinent history and identify patients at risk for breast cancer based on their personal history, family history, and breast imaging/biopsy history, if relevant. We also need to improve our discussions of supplemental screening for patients who have heterogeneously dense or extremely dense breast tissue. I sense that some ObGyns may rely heavily on the radiologist to suggest supplemental screening, but patients actually look to ObGyns as their providers to have this knowledge and give them direction.

Since I practice at a large academic medical center, I have the opportunity to refer patients to our Breast Cancer Genetics Program because I may be limited on time for counseling in the office and do not want to miss salient details. With all of the information I have ascertained about the patient, I am able to determine and encourage appropriate screening and assure insurance coverage for adjunctive breast MRI when appropriate.

\section{Consider how you order patients' screening to reduce barriers and cost}

\section{How would you suggest reducing barriers when referring patients for supplemental screening, such as MRI for high-risk women or ultrasound for those with dense breasts? Would you prefer it if such screening could be performed without additional script/referral? How does insurance coverage factor in?}

Dr. Etkin-Kramer: I would love for a screening mammogram with possible ultrasound, on one script, to be the norm. One of the centers that I work with accepts a script written this way. Further, when a patient receives screening at a freestanding facility as opposed to a hospital, the fee for the supplemental screening may be lower because they do not add on a facility fee.
Dr. Albright: We have an order in our electronic health record that allows for screening mammography but adds on diagnostic mammography/bilateral ultrasonography, if indicated by imaging. I am mostly ordering that option now for all of my screening patients; rarely have I had issues with insurance accepting that script. As for when ordering an MRI, I always try to ensure that I have done the patient's personal risk assessment and included that lifetime breast cancer risk on the order. If the risk is $20 \%$ or higher, I typically do not have any insurance coverage issues. If I am ordering MRI as supplemental screening, I typically order the "Fast MRI" protocol that our center offers. This order incurs a $\$ 299$ out-of-pocket cost for the patient. Any patient with heterogeneously or extremely dense breasts on mammography should have this option, but it requires patient education, discussion with the provider, and an additional cost. I definitely think that insurers need to consider covering supplemental screening, since breast density is reportable in a majority of the US states and will soon be the national standard.

\section{Pearls for guiding patients}

5. How do you discuss breast density and the need for supplemental screening with your patients?

Dr. Etkin-Kramer: I strongly feel that my patients need to know when a screening test has limited ability to do its job. This is the case with dense breasts. Visuals help; when discussing breast density, I like the images supplied by DenseBreast-info.org (FIGURE, page 24). I explain the two implications of dense tissue:

- First, dense tissue makes it harder to visualize cancers in the breast-the denser the breasts, the less likely the radiologist can pick up a cancer, so mammographic sensitivity for extremely dense breasts can be as low as $25 \%$ to $50 \%$.

- Second, high breast density adds to the risk of developing breast cancer. I explain that supplemental screening will pick up additional cancers in women with dense breasts. For example, breast ultrasound will 


\section{Figure Fatty vs dense breast tissue}

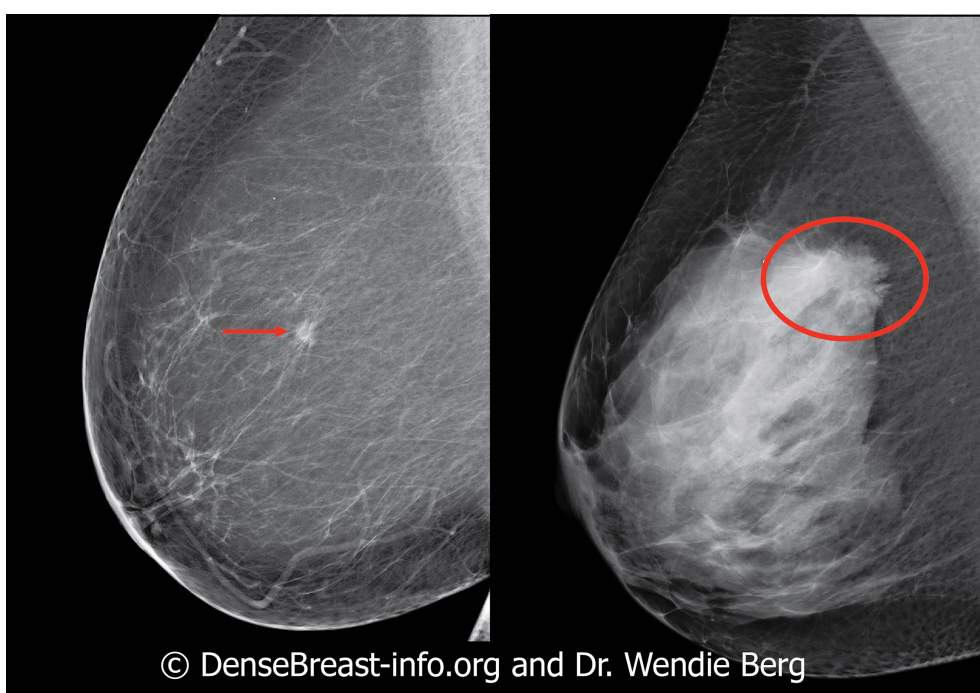

Cancers are difficult to detect in dense tissue (right) using mammography. Courtesy of DenseBreast-Info.org and Wendie Berg, MD, PhD.

\section{FAST}

TRACK

Patient

discussions

should include

the associated

risks of cancer

with dense

breasts pick up about 2-3/1000 additional breast cancers per year and MRI or molecular breast imaging (MBI) will pick up much more, perhaps 10/1000.

MRI is more invasive than an ultrasound and uses gadolinium, and MBI has more radiation. Supplemental screening is not endorsed by ACOG's most recent Committee Opinion from $2017 ;^{4}$ however, patients may choose to have it done. This is where shareddecision making is important.

I strongly recommend that all women's health care providers complete the CME course on the DenseBreast-info.org website. "Breast Density: Why It Matters" is a certified educational program for referring physicians that helps health care professionals learn about breast density, its associated risks, and how best to guide patients regarding breast cancer screening.

Dr. Albright: When I discuss breast density, I make sure that patients understand that their mammogram determines the density of their breast tissue. I review that in the higher density categories (heterogeneously dense or extremely dense), there is a higher risk of missing cancer, and that these categories are also associated with a higher risk of breast cancer. I also discuss the potential need for supplemental screening, for which my institution primarily offers Fast MRI. However, we can offer breast ultrasonography instead as an option, especially for those concerned about gadolinium exposure. Our center offers either of these supplemental screenings at a cost of $\$ 299$. I also review the lack of coverage for supplemental screening by some insurance carriers, as both providers and patients may need to advocate for insurer coverage of adjunct studies.

\section{Educational resources \\ 6. What reference materials, illustrations, or other tools do you use to educate your patients? \\ Dr. Etkin-Kramer: I frequently use handouts} printed from the DenseBreast-info.org website, and there is now a brand new patient fact sheet that I have just started using. I also have an example of breast density categories from fatty replaced to extremely dense on my computer, and I am putting it on a new smart board.

Dr. Albright: The extensive resources available at DenseBreast-info.org can improve both patient and provider knowledge of these important issues, so I suggest patients visit that website, and I use many of the images and visuals to help explain breast density. I even use the materials from the website for educating my resident trainees on breast health and screening.

\section{References}

1. Bharucha PP, Chiu KE, Francois FM, et al. Genetic testing and screening recommendations for patients with hereditary breast cancer. RadioGraphics. 2020;40:913-936.

2. Freedman AN, Yu B, Gail MH, et al. Benefit/risk assessment for breast cancer chemoprevention with raloxifene or tamoxifen for women age 50 years or older. J Clin Oncol. 2011;29:2327-2333.

3. Pruthi S, Heisey RE, Bevers TB. Chemoprevention for breast cancer. Ann Surg Oncol. 2015;22:3230-3235.

4. American College of Obstetricians and Gynecologists. Committee opinion no. 625: management of women with dense breasts diagnosed by mammography [published correction appears in Obstet Gynecol. 2016;127:166]. Obstet Gynecol. 2015;125(3):750-751. 


\section{MRI's role in breast cancer screening for childhood cancer survivors}

Nearly 16,000 children (up to age 19 years) face cancer-related treatment every year. ${ }^{1}$ For girls and young women, undergoing chest radiotherapy puts them at higher risk for secondary breast cancer. In fact, they have a 30\% chance of developing such cancer by age 50-a risk that is similar to women with a BRCA1 mutation. ${ }^{2}$ Therefore, current recommendations for breast cancer screening among those who have undergone childhood chest radiation ( $\geq 20 \mathrm{~Gy}$ ) are to begin annual mammography, with adjunct magnetic resonance imaging (MRI), at age 25 years (or 8 years after chest radiotherapy). ${ }^{3}$

To determine the benefits and risks of these recommendations, as well as of similar strategies, Yeh and colleagues performed simulation modeling using data from the Childhood Cancer Survivor Study and two CISNET (Cancer Intervention and Surveillance Modeling Network) models. ${ }^{4}$ For their study they targeted a cohort of female childhood cancer survivors having undergone chest radiotherapy and evaluated breast cancer screening with the following strategies:

- mammography plus MRI, starting at ages 25,30 , or 35 years and continuing to age 74

- MRI alone, starting at ages 25, 30, or 35 years and continuing to age 74 .

They found that both strategies reduced the risk of breast cancer in the targeted cohort but that screening beginning at the earliest ages prevented most deaths. No screening at all was associated with a $10 \%$ to $11 \%$ lifetime risk of breast cancer, but mammography plus MRI beginning at age 25 reduced that risk by $56 \%$ to $71 \%$ depending on the model. Screening with $\mathrm{MRI}$ alone reduced mortality risk by $56 \%$ to $62 \%$. When considering cost per qualityadjusted life-year gained, the researchers found that screening beginning at age 30 to be the most cost-effective. ${ }^{4}$

Yeh and colleagues addressed concerns with mammography and radiation. Although they said the associated amount of radiation exposure is small, the use of mammography in women younger than age 30 is controversial-and not recommended by the American Cancer Society or the National Comprehensive Cancer Network. ${ }^{5,6}$

Bottom line. Yeh and colleagues conclude that MRI screening, with or without mammography, beginning between the ages of 25 and 30 should be emphasized in screening guidelines. They note the importance of insurance coverage for MRI in those at risk for breast cancer due to childhood radiation exposure. ${ }^{4}$

\section{References}

1. National Cancer Institute. How common is cancer in children? https://www.cancer.gov/types/childhood-cancers/child-adolescentcancers-fact-sheet\#how-common-is-cancer-in-children. Accessed September 25, 2020.

2. Moskowitz CS, Chou JF, Wolden SL, et al. Breast cancer after chest radiation therapy for childhood cancer. J Clin Oncol. 2014;32:2217 2223.

3. Children's Oncology Group. Long-term follow-up guidelines for survivors of childhood, adolescent, and young adult cancers. http:// www.survivorshipguidelines.org/pdf/2018/COG_LTFU_Guidelines_v5.pdf. Accessed September 25, 2020.

4. Yeh JM, Lowry KP, Schechter CB, et al. Clinical benefits, harms, and cost-effectiveness of breast cancer screening for survivors of childhood cancer treated with chest radiation. Ann Intern Med. 2020;173:331-341.

5. Saslow D, Boetes C, Burke W, et al; American Cancer Society Breast Cancer Advisory Group. American Cancer Society guidelines for breast screening with MRI as an adjunct to mammography. CA Cancer J Clin. 2007;57:75-89.

6. National Comprehensive Cancer Network. NCCN Clinical Practice Guidelines in Oncology. Breast cancer screening and diagnosis version 1.2019. https://www.nccn.org/professionals/physician_gls/default.aspx. Accessed September 25, 2020. 